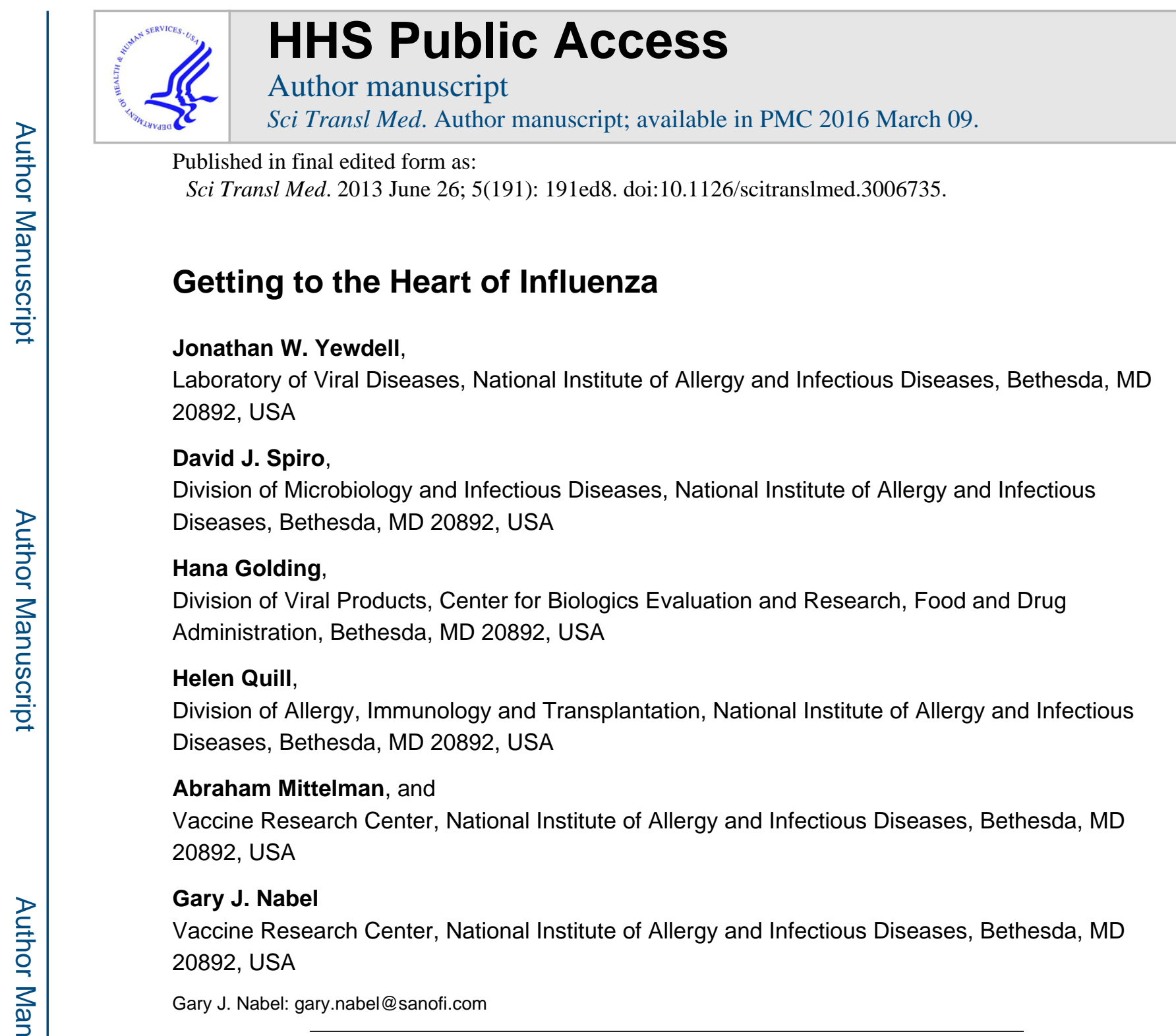

Advances in influenza virus biology have provided insight into mechanisms that can expand and enhance immune protection against diverse viral strains. To harness this knowledge and improve on the efficacy of current influenza vaccines, the U.S. Food and Drug

Administration and the National Institute of Allergy and Infectious Diseases of the U.S.

National Institutes of Health jointly sponsored a 2-day meeting (1) in which internationally recognized experts in influenza immunology, epidemiology, and vaccinology assembled with industry and government leaders (table S1) to discuss the development of universal influenza vaccines. Here, we summarize resulting recommendations for developing vaccines that provide durable protection against a class of viruses whose capacity for immune evasion necessitates yearly vaccination.

\footnotetext{
He is presently at Sanofi, 640 Memorial Drive, Cambridge, MA 02139, USA.

SUPPLEMENTARY MATERIALS

www.sciencetranslationalmedicine.org/cgi/content/full/5/191/191ed8/DC1

U.S. National Institute of Allergy and Infectious Diseases (NIAID) Resources for Researchers

Competing interests: The views expressed here represent those of the authors and not necessarily those of their institutions or of

other meeting participants and do not constitute an endorsement of a specific commercial product.
} 
In the United States, the annual burden of seasonal influenza can be as high as 49,000 deaths and more than 200,000 hospitalizations, resulting in $\$ 27$ billion in health costs alone and enormous economic costs in lost work time. The annual toll of these epidemics is estimated at 250,000 to 500,000 deaths worldwide (www.who.int/mediacentre/factsheets/2003/fs211/ en). Pandemic influenza occurred in 1918, 1957, 1968, and 2009 due to the introduction of novel influenza A viruses from both avian and swine reservoirs. Although the highly lethal avian H5N1 subtype has thus far not achieved effective human-to-human transmissibility this or other zoonotic influenza viruses continue to pose major pandemic threats (2).

Current vaccines are limited by influenza viruses' ability to evade humoral immunity. Antigenic drift describes accumulating amino acid substitutions in influenza virus surface glycoproteins that incrementally reduce the antiviral activity of antibodies specific for these glycoproteins. Antigenic shift refers to the introduction of new glycoproteins from zoonotic into human influenza viruses, as occurred with the recent introduction of the swine origin influenza virus in 2009, H1N1. These evolutionary changes abruptly alter glycoprotein antigenicity and eliminate the activity of most neutralizing antibodies (Abs) elicited by current vaccines $(3,4)$. Antigenic drift necessitates that the vaccine be updated frequentlyoften annually-to include new flu strains that match the predominant circulating variants. Antigenic shift requires the development of an entirely new vaccine, resulting in a period in which a large segment of the population remains vulnerable to infection while manufacturers scramble to produce the billions of doses necessary for worldwide immunization. A fundamentally new approach is needed to develop vaccines that circumvent antigenic drift and shift, as recommended for pandemic influenza virus preparedness (5).

Meeting participants devised the following plan for the development of broadly crossreactive influenza vaccines.

\section{CRITICAL NEEDS}

- Research that supports the rational design of immunogens that elicit long-lasting, high-affinity broadly protective Abs

- Development of easily performed assays capable of assessing potency of nextgeneration vaccines

- Animal models that elicit broadly neutralizing Abs and thus permit study of their role in protecting against infection

- Catalyzing of government and industry efforts to initiate large, potentially costly clinical trials for state-of-the-art vaccines devised from new research concepts

\section{STEPS THAT CAN BE TAKEN TO MEET THESE CHALLENGES}

- Definition of the B cell biology required to evolve and maintain high-affinity antibody responses to influenza vaccines as a function of age

- Elucidation of mechanisms of antiviral immunity conferred by broadly crossreactive Abs 
- Optimization of influenza virus immunogens capable of inducing broadly crossprotective immunity

- Development of animal models that better mimic the complexity and natural history of human immune responses to influenza virus infection and vaccine response

- Clinical studies to assess the immune response to and outcomes of influenza infection (including retrospective studies of existing specimens that can be correlated with known outcomes in previous trials)

\section{PRIORITIES FOR UNIVERSAL VACCINE DEVELOPMENT}

- Deciphering the requirements of universal influenza vaccine function

- Multiple small-scale human trials for promising candidate vaccines to catalyze public-private partnerships for large-scale clinical trials

- Controlled human infection models conducted relatively early in clinical development to demonstrate "proof of concept" of the advantages of drift-resistant vaccines (including increased breadth and duration of protection)

\section{DESIGN OF CLINICAL TRIALS FOR NEW INFLUENZA VACCINES SHOULD INCLUDE}

- A prospective case definition for influenza illness

- Inclusion of culture confirmation, viral typing, and antigenic characterization

- Immunogenicity evaluations in a substantial number of study participants, including measurement of cross-protective antibodies and cell-mediated immunity

- Characterization of the immune response in a clinical end point efficacy study as a possible way to extrapolate effectiveness in other populations

- Establishment of a reliable correlate of protection that, although not required for licensure, could greatly facilitate future influenza vaccine development

Considerable progress has been made in understanding influenza virus biology, and the time is ripe to harness the benefits of this research. By facilitating new consortia, public-private partnerships, enhanced clinical trial capacity, appropriate regulatory support, effective communication/coordination, and data sharing, it should be possible to more effectively prevent viral transmission and advance universal vaccines that address this pressing public health concern.

\section{Supplementary Material}

Refer to Web version on PubMed Central for supplementary material. 


\section{Biographies}

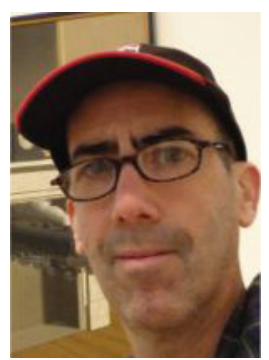

Jonathan W. Yewdell

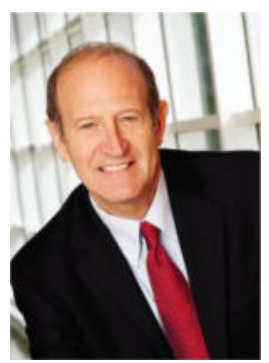

Gary J. Nabel

\section{References}

1. NIAID description of meeting. www.niaid.nih.gov/topics/Flu/Research/Pages/ReportsPlans.aspx

2. Lambert LC, Fauci AS. Influenza vaccines for the future. N Engl J Med. 2010; 363:2036-2044. [PubMed: 21083388]

3. Nabel GJ, Fauci AS. Induction of unnatural immunity: Prospects for a broadly protective universal influenza vaccine. Nat Med. 2010; 16:1389-1391. [PubMed: 21135852]

4. Wei CJ, Yassine HM, McTamney PM, Gall JG, Whittle JR, Boyington JC, Nabel GJ. Elicitation of broadly neutralizing influenza antibodies in animals with previous influenza exposure. Sci Transl Med. 2012; 4:147ra114.

5. Report to the President on reengineering the influenza vaccine production enterprise to meet the challenges of pandemic influenza. President's Council of Advisors on Science and Technology; Washington DC: 2010. www.whitehouse.gov/sites/default/files/microsites/ostp/PCAST-InfluenzaVaccinology-Report.pdf 